\title{
Etude expérimentale et comparative de filtre composite en argile, charbon actif et filtre moderne à partir des analyses des quatre échantillons des eaux de Dang de la région de l'Adamaoua.
}

\author{
Saint-Cyr LENGAYE ${ }^{1,2,3}$ Julien Bienvenu BOMANGAYEN SONGUELE ${ }^{1,3}$, Jean \\ M'BOLIGUIPA', Ruben M.MOUANGUE ${ }^{2}$ \\ ${ }^{1}$ Laboratoire d'Energétique Carnot, Université de Bangui (République Centrafricaine) \\ ${ }^{2}$ Laboratoire de Combustion et de Technologie Verte, Université de Ngaoundéré (Cameroun) \\ ${ }^{3}$ Laboratoire d'Hydrosciences Lavoisier, Chaire UNESCO sur la gestion de l'eau en République \\ Centrafricaine, Université de Bangui/ Faculté des Sciences (République Centrafricaine) \\ (lengayesaintcyr@gmail.com, jbbsonguele@yahoo.fr, jmboliguipa@yahoo.fr, r_mouangue@yahoo.fr)* \\ Corresponding Author; Ruben M. Mouangue, PO BOX. 455 Ngaoundéré Cameroun, Tel: +237 67746 \\ 10 06,r_mouangue@yahoo.fr /ruben.mouangue@univ-ndere.com.
}

\begin{abstract}
Résumé
Très souvent en zone rurale et milieu périurbain, on dénombre plusieurs sources d'eaux de puits, de sources naturelles et certains marigots qui sont exposées aux différentes intempéries et aux aléas de la nature. Ainsi, la consommation de ces eaux est à l'origine des maladies d'origine hydrique comme l'amibiase, la typhoïde, le cholera etc. Le présent travail vise à l'amélioration de l'hygiène et de l'environnement en zone rurale et dans certains quartiers périphériques de Dang et d'autres grandes villes de la région de l'Adamaoua. A ce titre, nous nous proposons de développer un filtre composite à base d'argile et de charbon actif issu des coques de noix de palme pour une large diffusion auprès des populations des zones rurales et périurbaines dans le but de réduire le taux des maladies d'origine hydrique et de faire une étude comparative des analyses physico-chimiques et microbiologiques des quatre échantillons et leur filtrat enfin d' évaluer le coût du procédé par rapport au filtre moderne. La méthode adoptée consiste à faire des échantillonnages d'argile et noix de coque de palme suivi d'un traitement physique pour obtenir des poudres d'argile, et puis, d'un traitement physico-chimique pour obtenir des poudres de charbons actifs par une activation thermique avec une température de 600 à $900^{\circ} \mathrm{C}$. Les résultats obtenus montrent que pour une eau non traitée de turbidité $2 \mathrm{NTU}$, ayant une forte présence $(+)$ de germes pathogènes (Escherichia colis), se voit cette caractéristique réduite après filtration à $1 \mathrm{NTU}$ avec une absence totale (/) de ces agents pathogènes.
\end{abstract}

Mots clés: Argile, charbon actif, potabilisation de l'eau, maladie hydrique, filtre composite 2

Received: $13 / 06 / 2019$
Accepted: $29 / 10 / 2019$
DOI: https//dx.doi.org/10.4314/jcas.v15i2.2
C The Authors. This work is licensed under the Creative Commons Attribution 4.0 International Licence. 


\begin{abstract}
Very often in rural and peri-urban areas, there are several sources of well water, natural springs and some marigots that are exposed to the various bad weather and natural hazards. Thus, the consumption of these waters is at the origin of waterborne diseases such as amoebiasis, typhoid, cholera, etc. This work aims to improve hygiene and the environment in rural areas and in some outlying areas of Dang and other major cities in the Adamawa region. As such, we propose to develop a composite filter based on clay and activated carbon from palm nut hulls for wide dissemination to rural and peri-urban populations in order to reduce the rate of dengue waterborne and to make a comparative study of the physicochemical and microbiological analyzes of the four samples and their filtrate finally to evaluate the cost of the process compared to the modern filter. The method adopted consists in making samples of clay and palm shell walnuts followed by a physical treatment to obtain clay powders, and then a physicochemical treatment to obtain activated carbon powders by a thermal activation with a temperature of 600 to $900{ }^{\circ} \mathrm{C}$. The results obtained show that for an untreated water of turbidity 2NTU, having a strong presence $(+)$ of pathogenic germs (Escherichia colis), see this characteristic reduced after filtration at a value of $1 \mathrm{NTU}$ with a total absence (/) of these pathogens.
\end{abstract}

Key words: Clay, activated carbon, water purification, water borne disease, composite filter 


\section{Introduction}

La technique de séparation à membrane est connue depuis un certain temps mais son développement reste récent (J. Desmod et Cains, 1999), et depuis les années 70 on retrouve ces techniques principalement dans les industries laitières, de boissons et traitement des eaux (V. Lenoble, 2003). Cette technique qui est un séparateur sélectif de particule, permet de purifier l'eau sans adjonction de produits chimiques (G. Mimanne et al., 2014) ; tout en conservant les propriétés minérales naturelles qu'elle contient ( $R$. Nguimalet et al, 2005). Ce procédé consomme relativement peu d'énergie et est sélectif (J.SONGUELE et al, 1994).Lorsqu'on effectue une filtration sur une membrane on obtient (D.G. Volpini, 1994) : -Le retentât, les particules fluides dont les diamètres supérieurs aux pores sont retenus par la membrane. -Le perméat, les particules de diamètres inférieurs qui passent aux travers de la membrane. Ces membranes souvent sont à base de céramique (Y. Lagabrielle, 1956) et depuis quelques décennies les techniques à membrane sont utilisées dans le domaine du traitement de l'eau potable (D. Samake, 2008). Cette technique utilise un gradient de pression pour faire passer l'eau au travers les pores des membranes (D. Samake, 2008). Des récentes études ont montré que l'argile était utilisée dans l'élaboration des supports macroporeux destinés à la fabrication de ces membranes (I. Papineau, 2013), En plus, il est reconnu que dans le traitement des eaux potables (H. El Haite, 2010), le charbon actif est souvent utilisé pour l'amélioration du goût et l'élimination de certaines substances indésirables et des micropolluants (G.P. Atheba, 2009), Malgré le fait que l'accès à l'eau potable et à l'assainissement soit reconnu comme droit de l'homme depuis 2010 , près de 3,5 milliards de personnes n'ont encore aujourd'hui d'autre choix que de boire une eau dangereuse pour leur santé (Youcef et al., 2014). 2,4 milliards de personnes disposent toujours de latrines qui sont à proximités de puits, soit $40 \%$ de la population mondiale. De ce fait, 2,6 millions de personnes meurent chaque année de maladies liées à l'eau, ce qui fait de l'eau insalubre une des premières cause de mortalité au monde (Y. Bessière, 2005), Eu égard à ces contraintes nous souhaitons concevoir un filtre composite à base d'argile et du charbon actif afin de réduire le taux de maladies liées à l'eau, pour cela nous avons envisagé de faire l'échantillonnages d'argile et coque de noix de palme ensuite mettre en œuvre le 4 filtre à eau pour une large diffusion auprès des populations qui n'ont pas d'accès à l'eau potable dans la ville de Ngaoundéré.

\section{2) Matériels et méthodes}

2.1) Liste du matériel Le matériel qui a été utilisé dans le cadre de ce travail est constitué de : - Un four Euro-term, dont la plage de température va de 0 à $1000^{\circ} \mathrm{C}$, - Une plaque chauffante, dont la plage de température va de 0 à $400^{\circ} \mathrm{C}$, - Un thermomètre infrarouge. - Une cocote minute, Un tamis normatifs, - Une boîte de pétrie, - Un turbidimètre, - Un pH-mètre, 2-2) Présentation et fonctionnement du filtre composite Le dispositif expérimental qui fait l'objet de cette étude est un filtre composite, constitué des matériaux locaux tels que, l'argile, le charbon actif et la farine de maïs. L'élaboration de ce procédé a été réalisé par de l'argile malaxé par les charbons actifs et la farine de maïs en utilisant la loi des proportions massiques suivi du mélange avec quelque volume d'eau pour obtenir une pâte malléable, suivi du moulage afin d'obtenir un modèle de filtre cylir

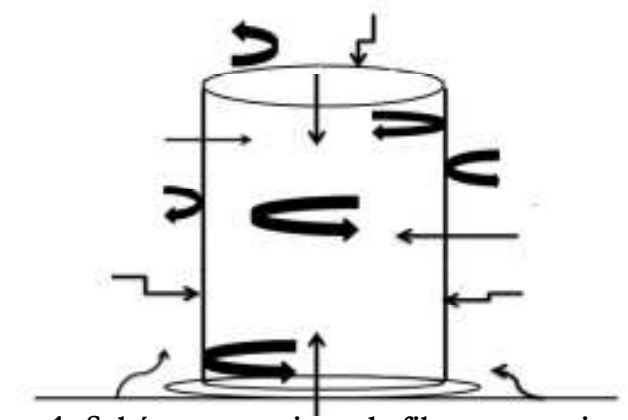

Figure 1: Schéma synoptique du filtre composite 
Par la suite le matériau a été séché en deux phases, une phase sous l'ombre pendant $72 \mathrm{~h}$ suivie d'une phase à l'air libre pour préparer le matériau à la cuisson. La cuisson est faite par un four électrique, elle est assurée d'abord un préchauffage du four à $100^{\circ} \mathrm{C}$ pendant $30 \mathrm{~min}$ pour passer de la température ambiante à une température élevée de l'ordre de $900^{\circ} \mathrm{C}$, lorsqu'on obtient la température de cuisson, cette dernière est maintenue pendant $2 \mathrm{~h}$ et finalement la phase de refroidissement où la température de la charge diminue progressivement. Le matériau composite étudié est constitué des produits à cuire. Cette charge est homogène car elle dépend de la granulométrie. La cuisson est assurée par un four électrique, la connaissance approfondie du fonctionnement thermique permet une maitrise de la phase de cuisson pour respecter la plage de température afin de garder les caractéristiques de l'argile sous l'effet de la température afin de mettre en œuvre les effets couplés de l'argile et les charbons actifs.

Le filtre composite se présente de la manière suivante, il est constitué d'une chambre de filtration composée d'un sceau de 10L et d'une membrane poreuse collée au milieu du sceau qui a un orifice de $3 \mathrm{~cm}$ de diamètre. Puis un autre sceau de $5 \mathrm{~L}$ où on place un récipient de récupération du filtrat. L'eau filtrée est versée dans la chambre de filtration et après deux heures (2h) temps, on obtient le filtrat.

2.3) Activation de la matière première Le procédé consiste à mettre en évidence les effets couplés de l'argile et le charbon actif qui sont des matériaux adsorbants efficaces dans le traitement des eaux. L'argile est un matériau adsorbant ses propriétés absorbantes sont connues depuis l'antiquité ; elle est utilisée dans plusieurs domaines, à savoir le domaine pharmaceutique, dans la poterie et les porcelaines. Les charbons actifs sont obtenus à partir des matières premières, par une pyrolyse pour enrichir le matériau en carbone et crée les premiers pores et suivi d'une activation chimique et physique afin de créer les surfaces absorbantes et augmenter son pouvoir adsorbant. La méthode d'activation adoptée se présente de la manière suivante, d'abord nous avons fait une imprégnation d'une masse de $4698 \mathrm{~g}$ des coques de noix de palme dans l'acide sulfurique, ce dernier est dilué dans $664 \mathrm{ml}$ d'eau distillée pendant $24 \mathrm{~h}$ afin de consolider les coques. Puis les coques imprégnées sont séchées pendant 72h. Ensuite nous avons pyrolisé les coques imprégnées dans un four électrique à $600^{\circ} \mathrm{C}$ pendant $2 \mathrm{~h}$ (Figure 2). L'activation thermique est retenue pour ce travail en utilisant l'activant acide phosphorique d'une concentration de $0.5 \mathrm{~mol} / \mathrm{L}$, puis cette solution est passée par une activation par vapeur dans une cocote minute portée sur une plaque chauffante à $400^{\circ} \mathrm{C}$ pendant 4 h (Figure 3). On obtient le charbon activé, ce dernier est lavé dans une eau pour se débarrasser des acides phosphoriques avant de les mettre à l'étuve à $88^{\circ} \mathrm{C}$ pendant $24 \mathrm{~h}$.

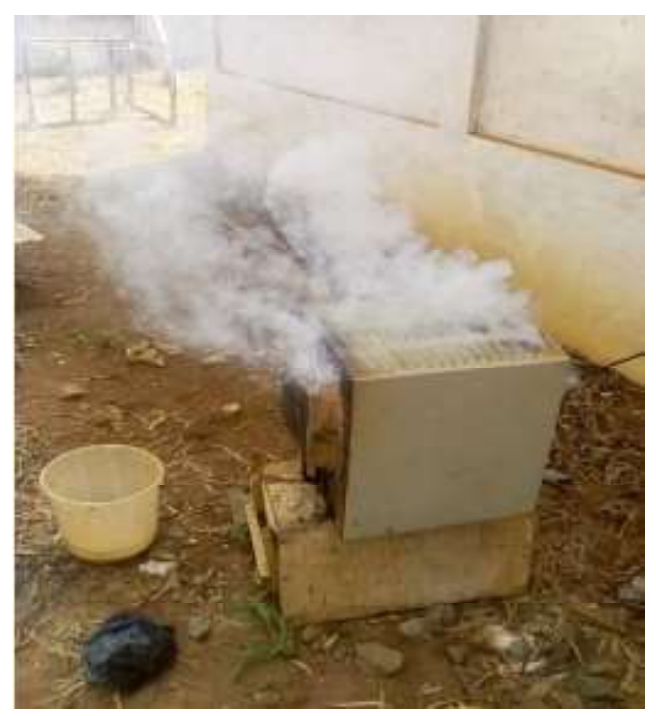

Figure 2 : Carbonisation des coques de noix de palme dans le four Euro therme 


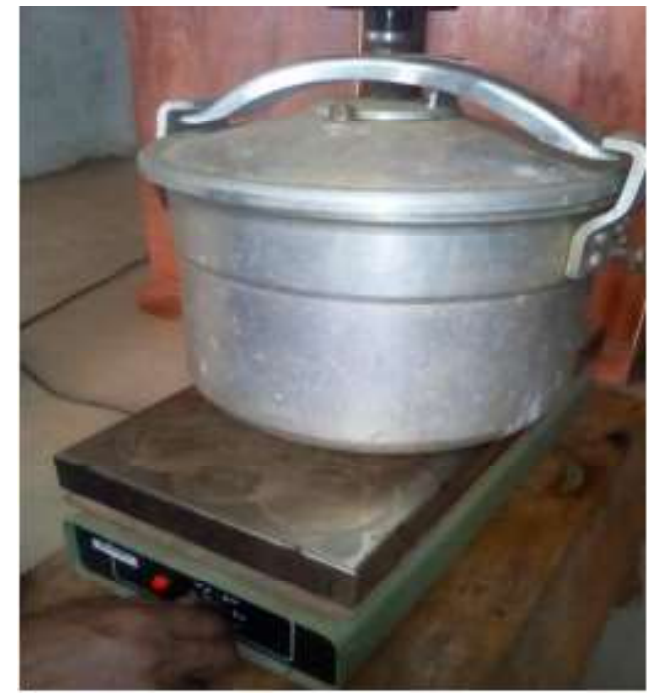

Figure 3 : Activation des charbons actifs par la méthode d'activation thermique

\section{4) Traitement thermique de la matière}

Le traitement thermique dans ce travail, est basé sur le séchage des matériaux à savoir le séchage d'argile et les coques de noix de palme. Ensuite vient le séchage du matériau composite après son élaboration. Ce séchage est caractérisé d'abord par un séchage sous l'ombre pendant $72 \mathrm{~h}$ (Figure 4) dans l'objectif d'éviter les fissures et un séchage à l'air libre (Figure 5) qui permet de réduire au maximum le taux d'humidité afin de préparer le matériau à la cuisson, la cuisson est faite à $900^{\circ} \mathrm{C}$ pendant 2 heures pour que les matières volatiles partent par les fumées afin de libérer des minuscules trous appelés des pores qui servent à l'écoulement de l'eau pendant la filtration. Le traitement thermique permet d'obtenir des améliorations dans deux directions : -Le changement de structure de la membrane, de phase plastique par le durcissement du matériau composite. - La libération des pores qui servent à l'écoulement poreux.

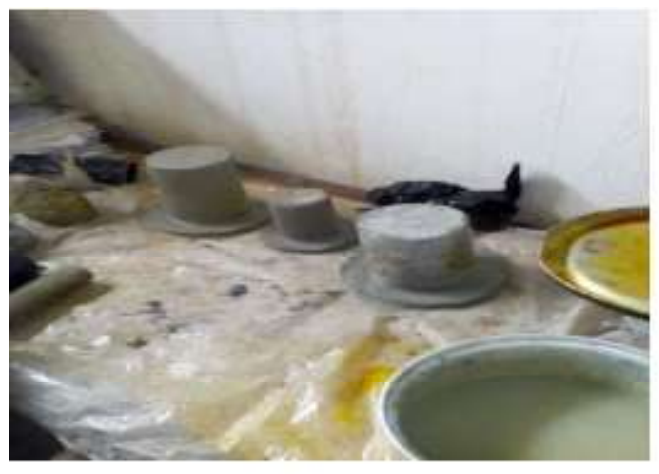

Figure 4: Phase de séchage sous l'ombre

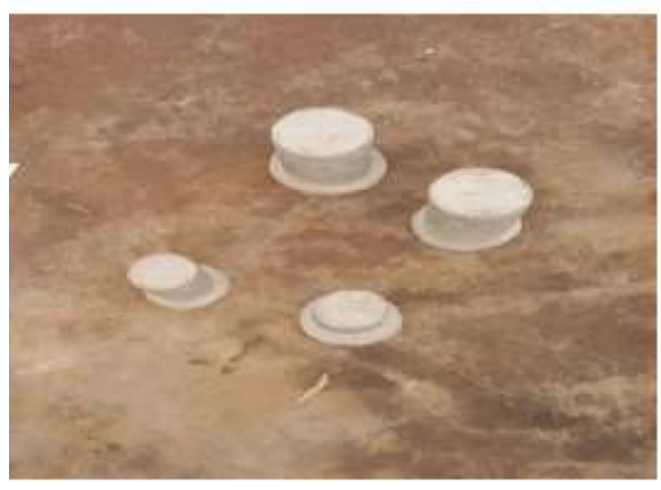

Figure 5: Phase de séchage à l'air libre

2.5) Prélèvement et conditionnement de quatre échantillons d'eau à traiter Le prélèvement d'un échantillon d'eau qui est une opération délicate à laquelle le plus grand soin doit être apporté ; il conditionne les résultats analytiques et l'interprétation qui en sera donnée. L'échantillon doit être homogène, représentatif et obtenu sans modifier les caractéristiques physicochimiques de l'eau (gaz dissous, matières en suspension, etc.) [23]. Pour notre 7 travail, après les prélèvements des quatre échantillons de l'eau à savoir : l'eau de puits (Figure 6), l'eau du lac artificiel de la ville de Dang (Figure 7), l'eau de forage (Figure 8) et enfin l'eau de robinet (Figure 9) de la société CDE (Camerounaise Des Eaux), ces échantillons sont conservés dans le réfrigérateur à $4{ }^{\circ} \mathrm{C}$. 


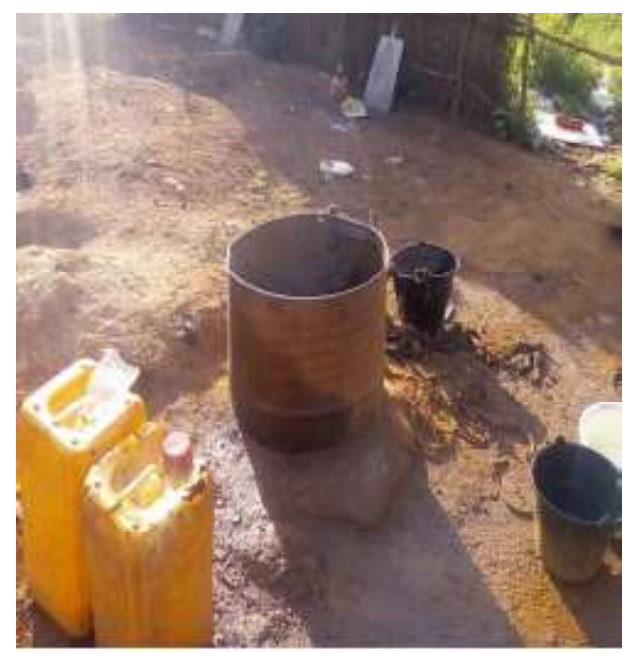

Figure 6 : Image du prélèvement de l'eau d'un puits

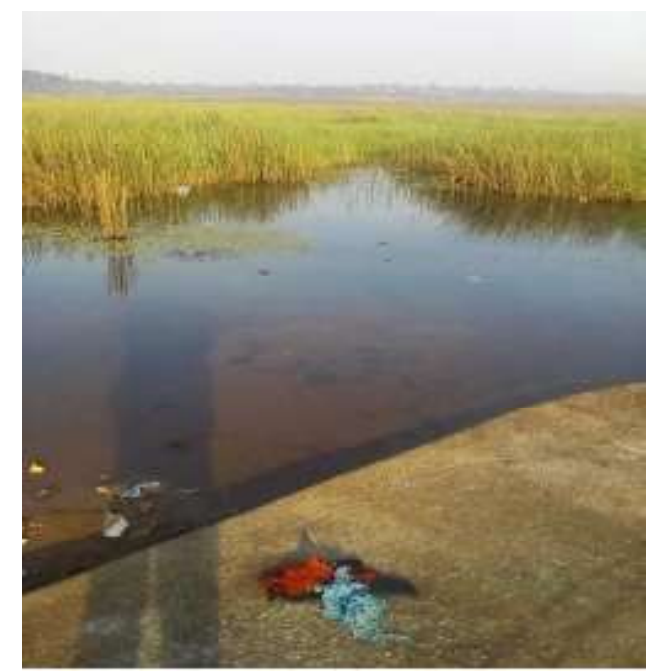

Figure 7: Image du prélèvement de l'eau du lac artificiel de Dang

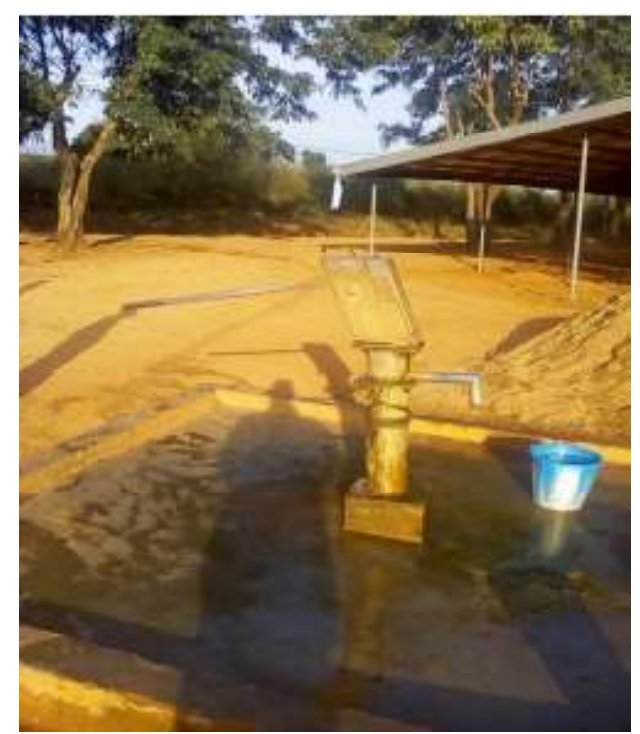

Figure 8: Image du prélèvement de l'eau du forage

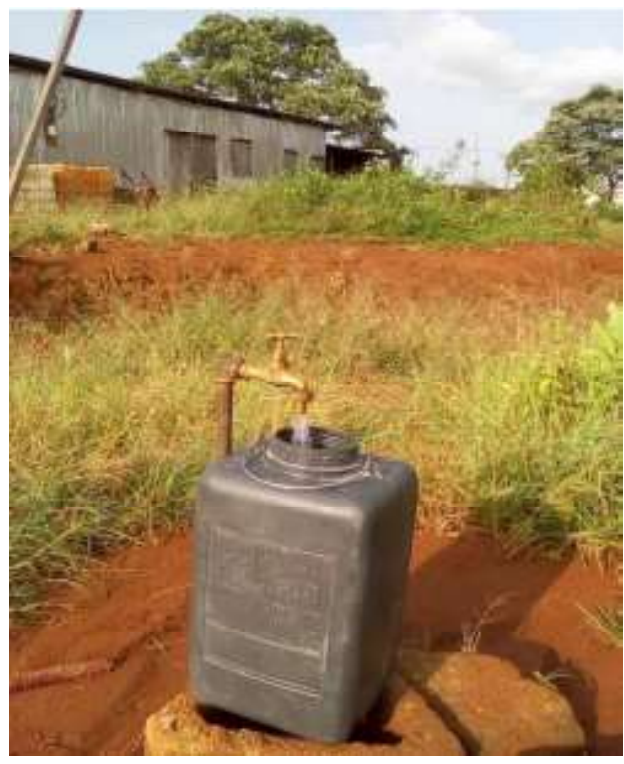

Figure 9: Image du prélèvement de l'eau du robinet

Au cours de la recherche des paramètres physicochimiques, deux paramètres sont évaluer à savoir la turbidité et le $\mathrm{pH}$ des quatre échantillons et leurs filtrats. Les matériels utilisés sont le turbidimètre et le $\mathrm{pH}$ mètre.

2.6) Analyse microbiologique des quatre échantillons d'eau prélevés

La salubrité microbiologique permet la détection des indicateurs microbiens de pollution fécale, à savoir les indicateurs des germes et des germes pathogènes. Il s'agit des organismes présents en grands nombres dans les matières fécales humaines ou animales. Leur détection signale un risque de contamination fécale (humaine ou animale) de la masse d'eau ou du réseau de distribution faisant l'objet du contrôle et, par conséquent, la présence possible des germes indicateurs. En général, les indicateurs microbiens ne sont pas eux-mêmes pathogènes chez l'humain. Pour évaluer la salubrité de l'eau potable, on utilise principalement les quatre indicateurs microbiens suivants : numération des bactéries coliformes totaux (CT), coliformes thermotolérants (CTT) et Escherichia coli (E. coli) et les Vibrios. Selon la méthode utilisée, il faut attendre 48 heures pour recevoir les résultats 
du laboratoire, ce qui signifie que l'eau est consommée avant que l'exploitant sache si elle peut être bue sans danger. Ces germes ont été déterminés par la méthode de filtration sur membrane telle que décrite par Rodier et al. (2009) en accord avec les normes telles que décrites par l'OMS en 2003. Cette méthode est très largement utilisée pour le dénombrement des germes de micro-organisme dans l'eau destinée à la consommation humaine. L'inconvénient majeur des indicateurs microbiens est la longueur du temps de culture des micro-organismes. Selon la méthode utilisée, il faut attendre 48 heures pour recevoir les résultats des analyses, ce qui signifie que l'eau est consommée avant que l'exploitant sache si elle peut être bue sans danger. Un autre inconvénient des analyses microbiennes réside dans leur coût par rapport aux contrôles physiques et chimiques effectués sur place. On distingue deux types de tests : les tests de présence-absence et les analyses quantitatives. Pour l'analyse par filtration sur membrane (FM), on commence par filtrer $100 \mathrm{ml}$ d'échantillon au travers d'une membrane, puis on dépose celle-ci sur un milieu de culture sélectif pour les coliformes ou pour Escherichia coli. À l'issue de l'incubation, on dénombre les colonies. L'analyse par filtration sur membrane (FM) est considérée comme la technique de référence.

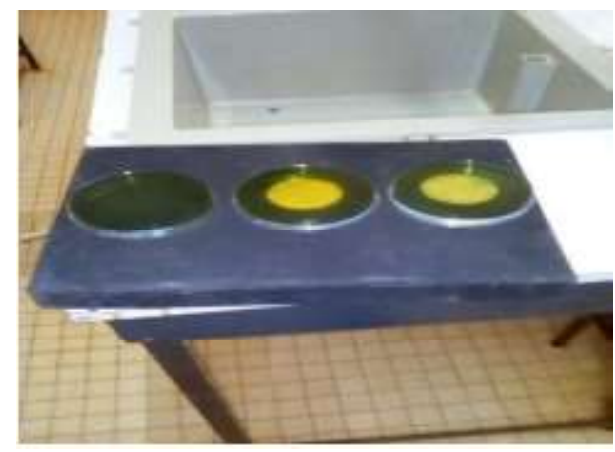

Figure 10: Image d'une boîte de pétrie 9

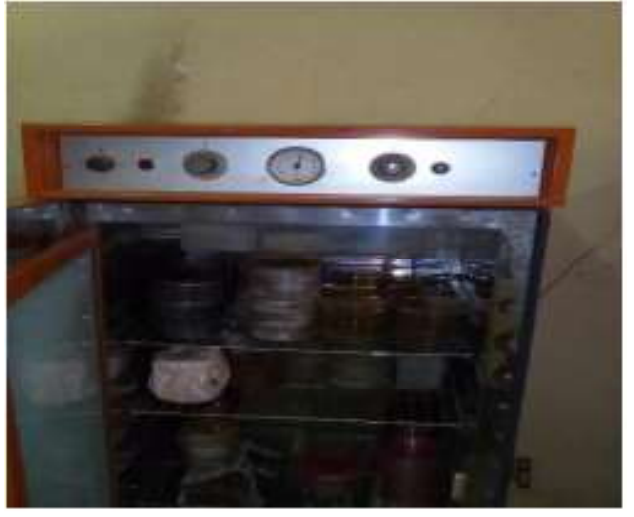

Figure 11: Image d'un incubateur

3. Résultats des travaux

Les résultats présentés dans ce travail, sont d'abord les résultats expérimentaux du profil de la température du matériau composite pendant le séchage ensuite les principaux résultats hydrodynamique du filtre composite et le filtre moderne, des paramètres physico-chimique et des analyses microbiologiques des quatre échantillons et leur filtrat de campagne de filtration des eaux de puits, du forage, du lac artificiel et l'eau du robinet de Camerounaise Des Eaux (CDE). Suivi d'une étude comparative des analyses faites par le filtre composite et le filtre moderne de marque chinoise suivi du coût.

3.1) Résultat des mesures durant le séchage, des températures sur les parois D'après la figure 12, on remarque que la montée de température moyenne varie en fonction de l'ensoleillement, dans un premier temps; la paroi extérieure 3 reçoit plus de chaleur cela montre une croissance exponentielle au point $\left(100 \mathrm{~min}, 55^{\circ} \mathrm{C}\right)$ suivi de la température intérieure, on remarque que la chaleur est conservée à l'intérieur du matériau composite. Ensuite quant aux parois extérieure 1 et extérieure 2, on constate que la paroi extérieure 1 est beaucoup frappée par les flux solaires et par rapport à la course du soleil l'autre paroi c'est-à-dire la paroi extérieure 2 sera plus frappée et ce phénomène est régit par la course du soleil. 


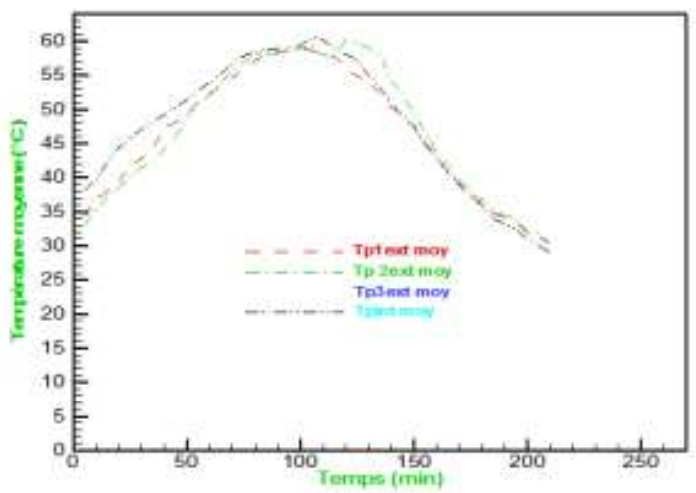

Figure 12: Profil de la température moyenne des parois du filtre composite pendant la campagne de séchage

3.2) Résultats de l'étude comparative de filtration à partir du filtre composite et d'un filtre moderne Quatre paramètres sont mis en exergue pendant cette étude hydrodynamique on peut citer entre autre le temps d'imbibition, le débit volumique, la durée de filtration et le volume de filtrat. Ces résultats sont consignés dans les tableaux 1 et 2 ci-après.

Cas du filtre composite : l'eau de forge utilisée par les populations de la ville de Dang (Tableau

1). Tableau 1 : Etude des paramètres hydrodynamiques du filtre composite.

\begin{tabular}{|c|c|c|c|c|}
\hline 10L & $\mathbf{5 L}$ & $\mathbf{2 L}$ & $\mathbf{1 L}$ & $\begin{array}{c}\text { Volume } \\
\text { Paramètre }\end{array}$ \\
\hline 1 & 2 & 3 & 5 & $\begin{array}{c}\text { Temps } \\
\text { d'imbibition } \\
\text { (min) }\end{array}$ \\
\hline 1,25 & 0,5 & 0,005 & 0 & $\begin{array}{c}\text { Débit } \\
\text { volumique }\end{array}$ \\
\hline 2 & 2 & 2 & 2 & $\begin{array}{c}\text { Durée de } \\
\text { filtration }\end{array}$ \\
\hline 2,5 & 1 & 0,03 & 0 & $\begin{array}{c}\text { Volume de } \\
\text { filtrat }\end{array}$ \\
\hline
\end{tabular}

Pour un volume de $1 \mathrm{~L}$ : On remarque que pour ce volume, on n'obtient pas de filtrat et le débit volumique est nul cela est due à la dimension du matériau composite qui est grande par rapport à ce volume.
Pour un volume de $2 \mathbf{L}$ : On obtient un faible volume de filtrat de $0,03 \mathrm{~L}$, suivi d'une faible valeur du débit $0,005 \mathrm{~L} / \mathrm{h}$, ces valeurs montrent l'augmentation du débit est fonction de la hauteur du volume d'échantillon d'eau pendant une durée de 2heures.

Pour un volume de 5L : On constate une légère augmentation de volume de filtrat de 1L, ensuite une croissance significative du débit volumique de $0,5 \mathrm{~L} / \mathrm{h}$ à la même durée.

Pour un volume de 10L : On assiste à une bonne valeur de volume de filtrat de $2,5 \mathrm{~L}$, suivi d'un bon débit volumique de valeur 1,25 L / h. Cette étude hydrodynamique, révèle que le débit d'eau est fonction de la hauteur de volume d'échantillon d'eau à traité suivi de la durée de filtration, donc une petite quantité de volume d'échantillon conduit à un faible débit volumique qui caractérise les paramètres hydrodynamique de filtration des eaux.

Cas du filtre moderne : 5L des différents échantillons d'eau ont été caractérisé (Tableau 2).

Tableau 2 : Etude des paramètres hydrodynamiques du filtre moderne

\begin{tabular}{|c|c|c|c|c|}
\hline $\begin{array}{c}\text { Eau du } \\
\text { robinet }\end{array}$ & $\begin{array}{c}\text { Eau de } \\
\text { puits }\end{array}$ & $\begin{array}{c}\text { Eau du } \\
\text { lac }\end{array}$ & $\begin{array}{c}\text { Eau du } \\
\text { forage }\end{array}$ & \\
\hline $\begin{array}{c}\text { Rien à } \\
\text { signaler }\end{array}$ & $\begin{array}{c}\text { Rien à } \\
\text { signaler }\end{array}$ & $\begin{array}{c}\text { Rien à } \\
\text { signaler }\end{array}$ & $\begin{array}{c}\text { Rien à } \\
\text { signaler }\end{array}$ & $\begin{array}{c}\text { Temps } \\
\text { d'imbibitio } \\
\text { n (min) }\end{array}$ \\
\hline 0.0952 & 0.071 & 0.071 & 0.0952 & $\begin{array}{c}\text { Débit vol. } \\
\text { (L/h) }\end{array}$ \\
\hline 21 & 21 & 21 & 21 & $\begin{array}{c}\text { Durée } \\
\text { filtration(h) }\end{array}$ \\
\hline 2 & 1.5 & 1.5 & 2 & $\begin{array}{c}\text { Vol. filtrat } \\
\text { (L) }\end{array}$ \\
\hline
\end{tabular}

Contrairement au filtre composite, d'abord le temps d'imbibition est différent c'est-àdire que la bougie ne libère pas le filtrat en forme de goutte, le débit d'eau du filtre moderne est très faible qui varie de 0.071 à $0.092 \mathrm{~L} / \mathrm{h}$ malgré la durée de 
filtration. Concernant la durée, le filtre moderne nécessite une longue durée pour que la bougie libère le filtrat, pour un volume nécessaire il faut au maximum une durée de $21 \mathrm{~h}$.

3.3) Analyse microbiologique des quatre échantillons d'eaux avant filtration

Pour ce qui est de l'évaluation de la qualité microbiologique de l'eau, (Tableau 3), les germes indicateurs de la qualité de l'eau et quelques germes pathogènes ont été recherchés et dénombrés par les méthodes de dilutions et de filtration sur membrane. Il s'agissait d'une part d'Escherichia coli, les coliformes fécaux, les coliformes totaux pour ce qui est des indicateurs de la qualité de l'eau et de Clostridium perfringens, Salmonelles et des Vibrios pour ce qui est des germes pathogènes. Ces germes ont été déterminés par la méthode de filtration sur membrane telle que décrite par Rodier et al. (2009) en accord avec les normes telles que décrites par l'OMS en (2003). Cette méthode est très largement utilisée pour le dénombrement des germes de micro-organisme dans l'eau destinée à la consommation humaine.

Tableau 3 : Résultats d'analyse des quatre échantillons d'eaux avant filtration (ND : non dénombrable, + : forte densité, / : absence totale)

\begin{tabular}{|c|c|c|c|c|c|c|c|c|}
\hline \multirow[b]{2}{*}{ Germes } & \multicolumn{4}{|c|}{$\mathrm{UFC} / \mathbf{m L}$} & \multicolumn{4}{|c|}{ UFC/100 mL } \\
\hline & 1 & 2 & 3 & 4 & 1 & 2 & 3 & 4 \\
\hline Coliformes totaux & $(1,3 \pm 0,2) \cdot 10^{4}$ & $(7,9 \pm 0,8) \cdot 10^{3}$ & $(6,8 \pm 0,4) \cdot 10^{3}$ & $(9,7 \pm 1,7) \cdot 10^{3}$ & ND & ND & ND & ND \\
\hline Coliformes fécaux & $(4,8 \pm 0,6) \cdot 10^{3}$ & 1 & $(4,5 \pm 1,3) \cdot 10^{3}$ & $(7,1 \pm 1,1) \cdot 10^{2}$ & ND & ND & ND & ND \\
\hline Escherichia coli & + & 1 & + & / & + & 1 & + & 1 \\
\hline $\begin{array}{l}\text { Streptocoques } \\
\text { fécaux }\end{array}$ & $\left(6,5 \pm 0,7.10^{3}\right.$ & I & $(1,3 \pm 0,3) \cdot 10^{3}$ & $(6,4 \pm 1,5) \cdot 10^{2}$ & ND & ND & ND & ND \\
\hline Vibrio sp. & $(2,7 \pm 0,5) \cdot 10^{3}$ & 7 & $(8,3 \pm 1,5) \cdot 10^{2}$ & & & & & \\
\hline $\begin{array}{l}\text { Clostridium sulfito- } \\
\text { réducteur }\end{array}$ & / & 1 & 1 & 7 & 7 & 7 & 7 & 1 \\
\hline Salmonella sp. & 7 & 7 & / & / & 1 & 1 & I & 1 \\
\hline
\end{tabular}

Ces résultats ont été ensuite comparés aux normes internationales de OMS et à la Directive 98/83/ CE du Conseil du 3 Novembre 1998 de l'Union Européenne (UE) relative à la qualité des eaux destinées à la consommation humaine en vue d'en tirer les conclusions appropriées.

3.4) Analyse microbiologique des quatre échantillons d'eaux après filtration par le filtre composite D'après le tableau 4, parmi les indicateurs microbiens présentés, l'Escherichia coli a une signification importante en santé publique. Cependant, en raison des limites des indicateurs utilisés, les contrôles microbiens prescrits permettent seulement d'indiquer qu'il pourrait y avoir des problèmes de qualité de l'eau. Un test positif pour l'Escherichia coli ne confirme pas que l'eau est contaminée par des agents pathogènes mais les systèmes de dénombrement ne ciblent pas la souche O157:H7 de l'Escherichia Coli. L'identification des agents pathogènes hydriques nécessite des méthodes et systèmes de dosage spécialisés. Par conséquent, un résultat positif pour l'Escherichia Coli n'indique pas nécessairement que l'eau contient un agent pathogène. La détection de l'Escherichia Coli peut également résulter d'une contamination lors de l'échantillonnage, auquel cas le test n'est pas représentatif de la masse d'eau contrôlée; cette hypothèse s'exclut en recueillant un autre échantillon pour confirmer la présence ou l'absence de l'indicateur. Ainsi, la détection d'autres germes pathogènes était donc nécessaire. 
Tableau 4: Résultats d'analyse des quatre échantillons d'eaux après filtration par le filtre composite (ND : non dénombrable, $+:$ forte densité, / : absence totale)

\begin{tabular}{|l|c|c|c|c|c|c|c|c|}
\hline & \multicolumn{3}{|c|}{ UFC/mL } & \multicolumn{4}{c|}{ UFC/100 $\mathbf{~ m L}$} \\
\hline Germes & $\mathbf{1}$ & $\mathbf{2}$ & $\mathbf{3}$ & $\mathbf{4}$ & $\mathbf{1}$ & $\mathbf{2}$ & $\mathbf{3}$ & $\mathbf{4}$ \\
\hline Coliformes totaux & $(9,6 \pm 2,2) \cdot 10^{4}$ & $(1,7 \pm 0,5) \cdot 10^{2}$ & $(7,8 \pm 1,3) \cdot 10^{3}$ & $/$ & $/$ & $/$ & $/$ & $5 \pm 3$ \\
\hline Coliformes fécaux & $(1,1 \pm 0,2) \cdot 10^{4}$ & $/$ & $(1,2 \pm 0,8) \cdot 10^{2}$ & $/$ & $/$ & $10 \pm 9$ & $8 \pm 5$ & $/$ \\
\hline Escherichia coli & + & + & + & $/$ & $/$ & $/$ & $/$ & $/$ \\
\hline Streptocoques fécaux & $/$ & $/$ & $/$ & $/$ & $/$ & $/$ & $/$ & $/$ \\
\hline Vibrio sp. & $/$ & $/$ & $/$ & $/$ & $/$ & $/$ & $/$ & $/$ \\
\hline $\begin{array}{l}\text { Clostridium sulfito- } \\
\text { réducteur }\end{array}$ & $/$ & $/$ & $/$ & $/$ & $/$ & $/$ & $/$ & $/$ \\
\hline Salmonella sp. & $/$ & $/$ & $/$ & $/$ & $/$ & $/$ & $/$ & $/$ \\
\hline
\end{tabular}

\section{5) Analyse microbiologique des quatre échantillons d'eaux filtrés par le filtre moderne}

Pour ce qui est de l'analyse des eaux traité au filtre moderne (Tableau 5), on note une absence complète des germes indicateurs de qualité et des germes pathogènes dans $1 \mathrm{ml}$ de chaque échantillon. Par ailleurs, après concentration de $100 \mathrm{ml}$ et ensemencement sur milieu de culture on note une prédominance de coliformes totaux de l'ordre de $(7,8 \pm 1,3) .102$ et de coliformes fécaux avec des valeurs inferieurs 100 UFC/ 100mL. Ces observations peuvent être liées à la charge microbienne globale des échantillons de départ, au temps de filtration long qui favoriserait une traversé des germes pour se retrouver dans le filtrat. 3.6) Analyse physico-chimique des quatre échantillons Quatre échantillons d'eaux fréquemment utilisé par les populations de Dang ; ont été caractérisés par les paramètres physicochimiques à savoir le $\mathrm{pH}$ et la turbidité des échantillons et les filtrats. Les résultats obtenus sont consignés dans les tableaux 6 et 7 ci-après. a) Avant le traitement Les résultats de $\mathrm{pH}$ de quatre échantillons, prouvent que les eaux du forage, du lac artificiel, de puits et l'eau du robinet sont quasiment basique car $5,48<\mathrm{pH}$

Tableau 5 : Résultats des quatre échantillons traités par le filtre moderne (ND : non dénombrable, + : forte densité, / :

$$
\text { absence totale) }
$$

\begin{tabular}{|c|c|c|c|c|c|c|c|c|}
\hline \multirow[b]{2}{*}{ Germes } & \multicolumn{4}{|c|}{ UFC/MI } & \multicolumn{4}{|c|}{ UFC/100 MI } \\
\hline & 1 & 2 & 3 & 4 & 1 & 2 & 3 & 4 \\
\hline Coliformes totaux & I & I & I & I & $(4,6 \pm 1,2) \cdot 10^{2}$ & $(2,7 \pm 0,5) \cdot 10^{2}$ & $(7,8 \pm 1,3) \cdot 10^{2}$ & $(4,6 \pm 1,2) \cdot 10^{2}$ \\
\hline Coliformes fécaux & 1 & 1 & 1 & 1 & 102 & I & 93 & 72 \\
\hline Escherichia coli & I & 1 & I & I & 1 & I & I & 1 \\
\hline Streptocoques fécaux & I & 1 & I & I & I & 1 & I & 1 \\
\hline Vibrio sp. & I & 1 & I & 1 & 1 & I & l & I \\
\hline Clostridium sulfito- réducteur & 1 & 1 & 1 & 1 & 1 & 1 & 1 & I \\
\hline Salmonella sp. & I & I & I & I & 1 & I & 1 & 1 \\
\hline
\end{tabular}

Quant à la turbidité, on relève que les quatre échantillons traités ne sont pas trouble c'est- à-dire il n'y a pas la présence des matières en suspension et toutes les valeurs obtenus sont conformes aux normes de la turbidité selon l'OMS $(<5 \mathrm{NTU})$. 
Tableau 6 : Résultats de l'analyse physicochimique des échantillons avant le traitement

\begin{tabular}{|c|c|c|}
\hline Paramètre & $\begin{array}{c}\text { Turbidité } \\
\text { (NTU) }\end{array}$ & pH \\
\hline Eau du forage & 01 & 7.74 \\
\hline Eau du lac & 01 & 6.18 \\
\hline Eau du puits & 01 & 5.48 \\
\hline Eau du robinet & 02 & 7,92 \\
\hline
\end{tabular}

\section{b) Après le traitement}

Les résultats susmentionnés, d'abord sont conformes aux normes admises du ph (6.5- 8.5, OMS ; 6.5-9.5, UE). Ces valeurs sont porteuses et montrent que les valeurs de $\mathrm{pH}$ des échantillons et des filtrats sont basiques donc les eaux traités ne sont pas acides. Quant à la turbidité, on constate d'abord que les échantillons prélevés ne sont pas trouble, cela montre l'absence des matières en suspension. Ensuite les valeurs de la turbidité sont conformes aux normes de la turbidité selon l'OMS qu'une eau potable doit avoir une turbidité inferieure à 5NTU.

Tableaux 7 : Résultats de l'analyse physico-chimique des échantillons après le traitement

\begin{tabular}{|c|c|c|}
\hline Paramètres & $\begin{array}{c}\text { Turbidité } \\
\text { (NTU) }\end{array}$ & Ph \\
\hline Eau du forage & 01 & 8.20 \\
\hline Eau du lac & 01 & 7.14 \\
\hline Eau du puits & 01 & 7.03 \\
\hline Eau du robinet & 01 & 9.45 \\
\hline
\end{tabular}

Les résultats susmentionnés, d'abord sont conformes aux normes admises du ph (6.5- 8.5, OMS ; 6.5-9.5, UE). Ces valeurs sont porteuses et montrent que les valeurs de $\mathrm{pH}$ des échantillons et des filtrats sont basiques donc les eaux traités ne sont pas acides. Quant à la turbidité, on constate d'abord que les échantillons prélevés ne sont pas trouble, cela montre l'absence des matières en suspension. Ensuite les valeurs de la turbidité sont conformes aux normes de la turbidité selon l'OMS qu'une eau potable doit avoir une turbidité inferieure à 5NTU.

\section{7) Etude comparative du taux d'humidité du matériau composite pendant les phases de séchage}

Cette partie consiste à évaluer le taux d'humidité du matériau composite, après son élaboration on pèse la masse humide puis nous effectuons le premier séchage sous l'ombre pour éviter que le matériau subisse des fissures. Puis le second séchage à l'air libre, consiste à réduire au maximum le taux d'humidité contenu dans le filtre composite. Les résultats obtenus pendant cette étude sont résumé dans le tableau 8 ci-dessous. Tableau 8 : Résultats du taux d'humidité du matériau composite pendant les deux séchages

\begin{tabular}{|c|c|c|c|}
\hline & $\begin{array}{c}\text { Taux } \\
\text { d'humidité }\end{array}$ & $\begin{array}{c}\text { Séchage à } \\
\text { l'air libre }\end{array}$ & $\begin{array}{c}\text { Séchage sous } \\
\text { l'ombre }\end{array}$ \\
\hline $\begin{array}{c}\text { Masse du } \\
\text { matériau } \\
\text { humide }\left(\mathbf{m}_{\mathbf{0}}\right)\end{array}$ & $2,66 \%$ & $1011,8 \mathrm{~g}$ & $1200 \mathrm{~g}$ \\
\hline $\begin{array}{c}\text { Masse du } \\
\text { matériau } \\
\text { séchée }\left(\mathbf{m}_{\mathbf{0}}\right)\end{array}$ & $90,69 \%$ & $917,7 \mathrm{~g}$ & $1105,9 \mathrm{~g}$ \\
\hline
\end{tabular}

Cette étude révèle que pendant le séchage sous l'ombre, une portion d'eau contenu dans le matériau son évaporer par l'action de l'air par un pourcentage de $2.66 \%$. Ensuite le séchage à l'air libre permet au matériau de libéré le maximum de quantité d'eau d'un pourcentage de 90,69\% 4) 
Discussion des résultats $\neg$ Comparaison sur le coût du filtre composite par rapport au filtre moderne Sur le plan économique, le filtre composite est moins cher d'un prix de 7500FCFA, par rapport au filtre moderne qui coûte 15000FCFA sur le marché (Figure 12). Au regard de ce qui précède, il est judicieux de valoriser le filtre composite et vulgariser sur toute l'étendue territorial pour aider les populations qui n'ont pas d'accès au réseau d'adduction d'eau potable et déplus ceux qui n'ont pas de moyen nécessaire de payer le filtre moderne.

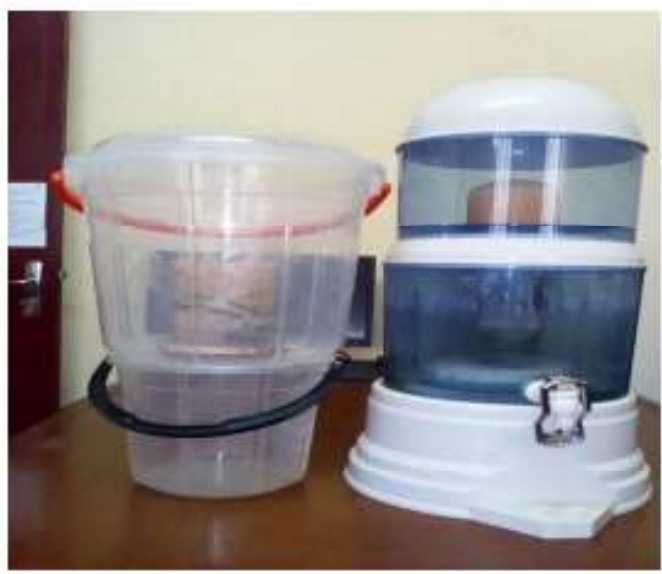

Figure 12 : Image du filtre composite (à gauche) et du filtre moderne (à droite)

\section{Comparaison sur les paramètres} hydrodynamiques

Le filtre composite est caractérisé par un débit volumique appréciable de 0,5 à $1,25 \mathrm{~L} / \mathrm{h}$, pendant une durée de $6 \mathrm{~h}$, on obtient un volume de filtrat qui a une plage de 1 à 2,5L. Par contre le filtre moderne, qui nécessite une longue durée de $21 \mathrm{~h}$ de filtration pour un faible débit volumique de 0,071 à $0,092 \mathrm{~L} / \mathrm{h}$ et un volume de filtrat de 2 , $5 \mathrm{~L}$.

\section{Comparaison sur les analyses microbiologiques}

D'un échantillon à un autre, des variations ont été constatées pour bon nombre des paramètres analysés. Les tableaux 3, 4 et 5 ci-dessus illustrent la variation des valeurs déterminées par paramètre dans les quatre (4) échantillons provenant de l'eau de forage, l'eau du lac artificiel, l'eau de puits de Dang et celle de la CDE par comparaison par le filtre composite et le filtre moderne. Les analyses faites à partir du filtre composite montrent une rétention considérable des germes indicateurs d'un nombre non dénombrable (ND) à un nombre dénombrable. $\mathrm{A}$ l'exemple des coliformes fécaux et totaux qui partent d'un nombre non dénombrable (ND) à une valeur dénombrable de 40 . Pour ce qui concerne des germes pathogènes tels que Clostridium perfringens, Salmonelles et Vibrios sp, nous constate également une diminution acceptable des densités de ces germes c'est-à-dire d'une densité non dénombrable (ND) à une densité dénombrable. C'est le cas des Vibrios sp. qui passent de $8,3 \pm 1,5$ x 102 à $60 \pm 5$. Cette rétention très significative des germes indicateurs et germes pathogènes révèle les effets couplés de l'argile et du charbon actif dans le traitement des eaux. Le rendement du filtre composite est de 95\% évalué à partir des concentrations initiales et finales.

Concernant le filtre moderne, les analyses des indicateurs des germes et les germes pathogènes de la qualité de l'eau susmentionnés montrent aussi un abattement raisonnable mais vue la durée de filtration qui est longue, certaines bactéries arrivent à traverser la bougie et contaminent le filtrat. Il est judicieux de ne pas laisser le filtrat pendant une longue durée avant de le consommer.

Le traitement des eaux à partir des argiles et charbons actifs et l'étude comparative du filtre composite et moderne dans le présent travail, illustre les effets de synergies des matériaux utilisés.

Les résultats des mesures des paramètres physicochimiques, les résultats obtenus sont similaires selon les travaux de Lenoble et Rodier $6,5<\mathrm{pH}$ 
Figure 13 : Phase expérimentale de filtration de l'eau de forage du quartier Bini-Dang (ville Universitaire)

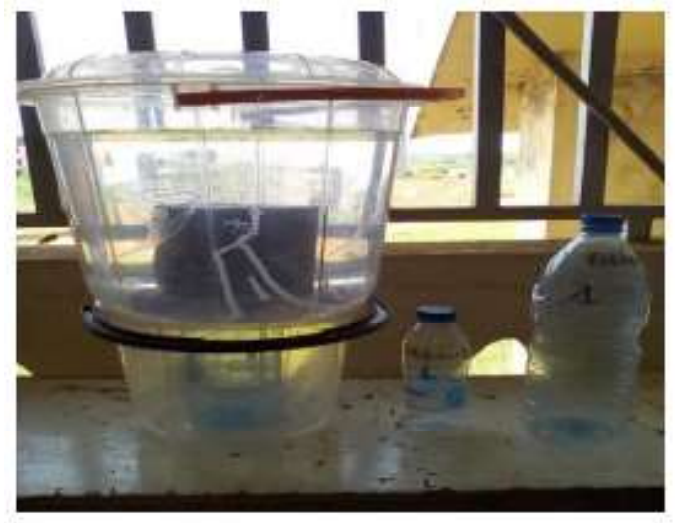

\section{5) Conclusion et perspectives}

Ce travail porte sur l'étude expérimentale et comparative du filtre composite et filtre moderne à partir des analyses des quatre échantillons d'eaux de Dang de la région de l'Adamaoua a permis de pouvoir à la communauté scientifique et a tout intéressé une compréhension globale du type et de la qualité de l'eau consommée par la population de Ngaoundéré en générale et en particulier celle de Dang à l'échelle locale. Il ressort des résultats que les eaux de forage, de puits, du lac artificiel et l'eau du robinet constituent les points d'approvisionnement en eau par la population pour tous les usagers domestiques (boisson, cuisine, lessive, baignade).

Sur le plan qualitatif, cette étude a montré que la quasi-totalité des paramètres physico-chimiques analysés sont conformes aux normes de l'OMS et de l'UE. En effet, sur les deux (2) paramètres physico-chimiques qui ont été considérés dans le cadre de ce travail, les deux (2) se révèlent conformes aux normes de l'Organisation Mondiales de la Santé (OMS), à savoir le pH $(5,48<\mathrm{pH}<7.92)$ suivi de la turbidité qui a une plage de 01 à 02NTU. Concernant les analyses microbiologiques, les analyses des germes indicateurs tels que les coliformes fécaux, totaux d'Escherichia colis montrent une réduction considérable des densités des germes indicateurs allant d'une densité non dénombrable (ND) à une densité dénombrable de 40. Concernant les germes pathogènes, les Clostridium perfringens, Salmonelles et des Vibrios sp, on note un abattement des densités des germes pathogènes de non dénombrable (ND) à une absence (/) mais certains germes pathogènes tels que les Vibrios sp on remarque une rétention dans certains échantillons de $141 \pm 4$ à $60 \pm 5$, cette étude nous emmène à un rendement de $95 \%$ du taux d'abattement des germes indicateurs et des germes pathogènes de la qualité de l'eau.

Toutefois, sur la base de ces constatations, des mesures urgentes incluant la population locale, les organisations communautaires de base ainsi que les agents de santé et d'hygiène dans la perspective de prévenir la population des risques de santé encourus par la consommation de ces eaux. En ce sens, il serait important :

* d'entreprendre une campagne de sensibilisation et de formation sur l'hygiène publique et les opérations de traitement de l'eau en vue d'éliminer les micro-organismes qui y sont présents ;

* d'entreprendre un programme de construction de latrines familiales dans tous les secteurs de Dang afin de réduire les risques de contamination hydro-fécale due à la défécation à l'air libre.

* de mobiliser les communautés de Dang à l'appropriation de la problématique de la gestion de l'environnement pour réduire la vulnérabilité des ressources en eau aux foyers de pollution.

\section{Remerciements}

Les auteurs souhaitent remercier le ministère Français des affaires étrangères représentées par le Service de la Coopération et de l'Action Culturelle (SCAC) de l'ambassade de France de Bangui, du Laboratoire de Combustion et de Technologie Verte de l'Université de Ngaoundéré, du Laboratoire d'Energétique Carnot et ainsi que le Laboratoire d' Hydro science Lavoisier pour la carbonisation des coques, l'activation du charbon actif et les analyses physico-chimiques et microbiologiques de la potabilité de l'eau. 


\section{Références}

1. Atheba. (2009). Traitement des eaux par action combinée de la photocatalyse solaire et de l'adsorption sur le charbon actif : conception et réalisation du procédé, pp : 1-192. Thèse, Université Paul Verlaine, Metz.

2. Balli et al. (2014). Etude des échanges thermiques dans un four à gaz pour la cuisson des produits céramiques, 19 Journal Verres, Céramiques et Composite, Vol.3.N²

3. Bessière.(2005). Filtration frontale sur membrane : mise en évidence du volume critique pour l'anticipation et le contrôle du colmata e, pp : 205, Thèse de Doctorat, Université Paul Sabatier

4. Coli et al. (2015). Maîtrise du risque lié à Vibrio parahaemolyticiens dans les produits halieutique destinés à l'exporation : cas d'une usine Sénégalaise de pêche, pp : 317-328, Journal Biotechnol. Agron.Soc Environ

5. Desmond et Cains. (1998). Filtration sur membrane, Article relative à la technique membranaire. Vol : 1- 8 ; pp : 20-30

6. Disa et al. (2014). Traitement des eaux de consommation par filtration lente sur sable à plusieurs étape, Vol : 27, n³, Université de Québec-INRS-Eau, Terre et environnement.

7. Ernesto. (2015). Préparation de charbon actif à partir de coques de noix de palmier à huile pour la récupération d'or et le traitement d'effluents cyanures, pp : 364, Thèse de Doctorat, Université catholique de Louvain.

8. Eddaba. (2011). Evaluation de la contamination bactériologique des eaux usées des stations d'épurations du grand GADIR : isolement, caractéristiques moléculaires et antibiorésistances des espèces, pp : 146, Thèse de Doctorat, Université IBN Zhr.

9. François. (1982). Traitement des eaux par électrocoagulation, énergie électrique et génie chimique. Université de Toulouse.

10. Ghannoum. (2014). Synthèse et caractérisation de matériau magnétiques pour l'adsorption de polluants présent dans les eaux, pp : 245 , Thèse de Doctorat, Université Pierre Marie Curie.

11. Hakima. (2010). Traitement des eaux usées par les réservoirs opérationnels et réutilisation pour l'irrigation, pp : 205, Thèse de Doctorat, Ecole Nationale Supérieure de Mines.

12. Lagabrielle. (1956). Membrane céramique d'ultrafiltration frontale, 12ème édition Vol : 301302

13. Lenoble. (2003). Elimination de l'Arsenic pour la préparation d'eau potable : oxydation chimique et adsorption sur substrats solides innovants, Thèse de Doctorat, thèse $\mathrm{n}^{\circ} 2, \mathrm{pp}: 1$ 198

14. Lengaye et al. (2019). Experimental and numerical study of composite filter based on Bangui clay and hull o palm : application to the purification of water, Journal of Taibah University for Science, Vol.13, NO.1 258-265

15. Leguary. (2005). Filtration au moyen du charbon actif, pp : 30-35 20

16. Manal. (2015). Elimination du zinc par adsorption par adsorption et par coagulation floculation, pp : 148, Thèse de Doctorat, Université Mohamed Khider Briska

17. Mimanne. (2014). Etude de l'adsorption des métaux lourds ( $\mathrm{Pb}$ et $\mathrm{Cd}$ ) en solution aqueuse sur 
charbon actif et montmorillonite sodée de l'Ouest Alger en pp : 1298 -1307, Journal Mater. Environ Science.

18. Maazouzi et al. (1960). Etude de procédé de filtration sur sable de la région de Bechur en prétraitement de l'eau potable, Vol : 15-20

19. Papineau. (2013). Influence des variations de qualité d'eau brute et du vieillissement des matériaux filtrants sur la performance de la filtration granulaire. Thèse de Doctorat, Ecole Polytechnique de Montré 1, pp : 183

20. Pialy. (2009). Etude de quelques matériaux argileux du site de Lembo (Cameroun) : minéralogie, comportement au filtrage et analyse des proportions d'élasticité, pp : 147, Thèse de Doctorat, Université de Limoges
21. Ralphi et al. (1995). Etude expérimentale des écoulements darcéens à travers un lit de fibres rigides empilées aléatoirement : influence de porosité. Journal Physiques I, pp : 1739-1756

22. Rodier et al. (2009). Analyse de l'eau. Collection Dunod.

23. Samake. (2003). Traitement des eaux usées de tannerie à l'aide de matériau à base d'argile, pp : 168, Thèse, Université Joseph Fourier de Grenoble et Université de Bamoko. 